\title{
Field-Induced Transitions in Nematic Liquid Crystals
}

\author{
A. M. Korostil, M. M. Krupa, D. O. Deretcha, Yu. B. Skyrta \\ Department of Magnetic Mesoscopic Materials and Nanocrystalline Nanostructures, Institute of \\ Magnetism of NAS of Ukraine and MES of Ukraine, Kyiv, Ukraine \\ E-mail: korostilandrii@gmail.com
}

Abstract: Magnetic-induced molecular orientational transitions in nematic liquid crystals doped by ferromagnetic nanoparticles have studied. On the basis of a microscopic approach of tensor operators it is sequentially considered the influence of the ferromagnetic dopes on the sensitivity of the orientational structure and the optical activity of the nematic liquid crystals.

Keywords: nematic liquid crystals; ferromagnetic nanoparticles; field-iduced molecular ordering; optical activity.

\section{INTRODUCTION}

Nematic liquid crystals, as systems of spatially non-localized and interconnected anisotropic molecules with a collective orientational ordering, are of considerable scientific interest because of the unusual nature of physical properties and important practical interest as functional materials, in particular for optical modulation devices and liquid crystal displays (see [1]). They are characterized by strong dependence of optical properties on the structure of molecular orientation ordering and abnormal optical activity with frequency-dependent polarization twisting of electromagnetic radiation, on orders of magnitude higher than in other condensed systems. Caused by fluidity, the susceptibility of the molecular orientational structure to the external fields provides the nematic liquid crystals the properties of the field controllability of their optical characteristics and modulation of electromagnetic radiation.

The problem of simulating the physical properties of nematic liquid crystals to identify their new functionalities and expand the scope of their application is topical. In particular, it concerns the enhancement of optical response parameters and minimization of their sizes.

The orientational molecular ordering of liquid crystals is characterized by their sensitivity to external fields, so-called threshold fields causing orientational transitions of liquid crystals. The 
latter is determined by the interphase interaction with solid surfaces, including confining surfaces and the surfaces of impurities. A significant increase of the magnetic sensitivity of liquid crystals is an urgent problem, the solution of which greatly expands the possibilities of creating new liquid crystal devices.

Perspectives of increasing of the magnetic sensitivity of nematic liquid crystals is related to using indirect interaction of a magnetic field with anisotropic molecules through impurity magnetic nanoparticles [1--3]. Corresponding colloidal systems are nematic crystals with impurity magnetic nanoparticles of small concentration have low threshold magnetic fields of the order of tens of Gauss [2]. Such small magnetic fields are not sufficient to reorient anisotropic molecules in pure nematic liquid crystals. However, they are sufficient for the rotation of the magnetic moments of the nanoparticles transmitting to anisotropic molecules of liquid crystal. In so doing, it is assumed the strong enough coupling of the magnetic nanoparticles with anisotropic molecules that provides the magnetic-induced elastic deformation of the orientational ordering leading to the orientational transition.

A self-consistent description of the molecular dynamics of the nematic liquid crystals involves the representation of their free energy through the finite moments of the orientational variables with respect to an orientational equilibrium distribution function. This results in the representation of tensorial parameters of orientational ordering [4]. Corresponding approach is based on microscopic description of the intermolecular interaction and the self-consistence derivation of the tensorial representation of the total free energy.

\section{TENSORIAL MODEL OF THE FREE ENERGY}

The orientational structure of nematic crystals is determined by the angular positions of the anisotropy molecule axes described by a orientational variable $\boldsymbol{m}$ relative to the director $\boldsymbol{n}$ of the middle direction that is expressed through the scalar product $\boldsymbol{n} \cdot \boldsymbol{m}$. For the distribution function $f(\boldsymbol{x}, \boldsymbol{m})$ of the orientational ordering of anisotropic molecules the free energy is described by the general expression [4]

$$
\begin{aligned}
& F[f]=\frac{1}{\beta} \int_{\Omega} \int_{S^{2}} f(\boldsymbol{x}, \boldsymbol{m})(f(\boldsymbol{x}, \boldsymbol{m})-1) \\
& \left.+\frac{1}{2 \beta} \bar{U}(\boldsymbol{x}, \boldsymbol{m}) f(\boldsymbol{x}, \boldsymbol{m})\right] d \boldsymbol{x} d \boldsymbol{m},
\end{aligned}
$$


where $\beta=k_{B} T$ ( $k_{B}$ is the Boltzmann constant, $T$ is the absolute temperature), and $\bar{U}$ is the meanfield interaction potential:

$$
\bar{U}(\boldsymbol{x}, \boldsymbol{m})=\beta \int_{\Omega} \int_{S^{2}} G\left(\boldsymbol{x}, \boldsymbol{m} ; \boldsymbol{x}^{\prime}, \boldsymbol{m}^{\prime}\right) f\left(\boldsymbol{x}^{\prime}, \boldsymbol{m}^{\prime}\right) d \boldsymbol{x}^{\prime} d \boldsymbol{m}^{\prime} .
$$

Here $G\left(\boldsymbol{x}, \boldsymbol{m} ; \boldsymbol{x}^{\prime}, \boldsymbol{m}^{\prime}\right)$ is the interaction kernel between two molecules in the configuration $(\boldsymbol{x}, \boldsymbol{m})$ and $\left(\boldsymbol{x}^{\prime}, \boldsymbol{m}^{\prime}\right)$. The assumption that states of the liquid crystal are very closed to the equilibrium, in the case when the concentration $c(x)=\int_{S^{2}} f(\boldsymbol{x}, \boldsymbol{m}) d \boldsymbol{m}$ is small the second virial expansion is valid and the corresponding free energy approximation can be written as

$$
\begin{gathered}
F[f]=\beta \int f(\boldsymbol{x}, \boldsymbol{m}) f(\boldsymbol{x}, \boldsymbol{m}) d \boldsymbol{x} d \boldsymbol{m}+\frac{1}{2} \int f(\boldsymbol{x}, \boldsymbol{m}) \\
\times G\left(\boldsymbol{x}, \boldsymbol{m} ; \boldsymbol{x}^{\prime}, \boldsymbol{m}^{\prime}\right) f\left(\boldsymbol{x}^{\prime}, \boldsymbol{m}^{\prime}\right) d \boldsymbol{x} d \boldsymbol{m} d \boldsymbol{x}^{\prime} d \boldsymbol{m}^{\prime} .
\end{gathered}
$$

Here the kernel function is defined as the classical expression for the second virial coefficient:

$$
G\left(\boldsymbol{r}, \boldsymbol{m}, \boldsymbol{m}^{\prime}\right)=1-\exp \left(-U\left(\boldsymbol{r}, \boldsymbol{m}, \boldsymbol{m}^{\prime}\right) / \beta\right),
$$

where $\boldsymbol{r}=\boldsymbol{x}-\boldsymbol{x}^{\prime}$ and $U$ is an intermolecular potential, which is determined by the shape of molecules and the interaction potential.

The approximation of the orientational distribution function $f\left(\boldsymbol{x}^{\prime}, \boldsymbol{m}^{\prime}\right)$ by its finite-order Taylor expansion series with respect to $\boldsymbol{x}^{\prime}$ at $\boldsymbol{x}$ and the same finite-order Taylor expansion series with respect to $\boldsymbol{m}^{\prime}$ at $\boldsymbol{m}$ result in the representation of the free energy through the $k$ th order symmetric tensors

$$
Q_{k}[f]=\int_{S^{2}} \Xi_{k}(\boldsymbol{m}) f(\boldsymbol{m}) d \boldsymbol{m} .
$$

Here $\Xi_{k}(\boldsymbol{m})$ is the $k$ th order symmetric tensor defined on the unite sphere. In so doing, it is used usage so-called the Bingham closure [4]

$$
f(\boldsymbol{x}, \boldsymbol{m})=c(\boldsymbol{x}) \frac{\exp \left(B_{Q}(\boldsymbol{x}): \boldsymbol{m m}\right)}{\int_{S^{2}}\left(B_{Q}(\boldsymbol{x}): \boldsymbol{m} \boldsymbol{m}\right) d \boldsymbol{m}},
$$

where $B_{Q}(\boldsymbol{x})$ is a symmetric matrix.

As the result, the total free energy is expressed through the $Q$-tensors and the molecular density $c(x)$ and their derivatives by the $\operatorname{sum} F_{e}[c, Q]=F_{b}+F_{e l 2}+F_{e l 4}$ expressed in terms of the introduced quantities $Q^{c}=c(\boldsymbol{x}) Q$ and $Q_{k}^{c}=c(\boldsymbol{x}) Q_{k}^{c}$, where $Q$ is the second moment of the orientational variable . The first term

$$
\begin{gathered}
F_{b}=\frac{1}{\beta} \int_{\Omega}\left[c\left(\ln c+B_{Q}: Q-\ln Z(\boldsymbol{x})\right)\right. \\
\left.+2\left(A_{1} c^{2}-A_{2}\left|Q^{c}\right|^{2}-A_{3}\left|Q_{4}^{c}\right|^{2}\right)\right] d \boldsymbol{x}
\end{gathered}
$$


is the bulk energy which contains the entropy and the quadratic terms of the order parameters. The second term

$$
\begin{gathered}
F_{e l 2}=\frac{1}{2 \beta} \int\left[-G_{\Omega}|\nabla c|^{2}+G_{2}\left|\nabla Q^{c}\right|^{2}+G_{3} \partial_{i} Q_{i k}^{c} \partial_{j} Q_{j k}^{c}-G_{4} \partial_{i} Q_{i j}^{c} \partial_{j} c\right. \\
\left.+G_{5}\left|\nabla Q_{4}^{c}\right|^{2}+G_{6} \partial_{i} Q_{4 i k l m}^{c} \partial_{j} Q_{4 j k l m}^{c}+G_{7} \partial_{i} Q_{4 i j k l}^{c} \partial_{j} Q_{k}^{c}\right] d \boldsymbol{x}
\end{gathered}
$$

is the second order elastic energy containing the derivative terms of the order parameters. The third term

$$
\begin{array}{r}
F_{e l 4}=\frac{1}{2 \beta} \int_{\Omega}\left[H_{1}\left|\nabla^{2} c\right|^{2}+H_{2}\left|\nabla^{2} Q^{c}\right|^{2}\right. \\
\left.+H_{3} \partial_{i j} Q_{i j}^{c} \partial_{k l} Q_{k l}^{c}+H_{4} \partial_{i k} Q_{i p}^{c} \partial_{j k} Q_{j p}^{c}\right]
\end{array}
$$

is the second order elastic energy containing the second order derivative terms of the order parameters.

The molecular orientational ordering in the liquid crystals is determined by the condition of the constrained minimum of the total free energy functional $F_{t}$, that is expressed by zero value of its variation, i.e. $\delta F=0$ at interface conditions with respect to order parameters. The latter is reduced to the equations describing static and dynamic states of liquid crystals.

\section{TRESHOLD MAGNETIC FIELD}

Conditions of the equilibrium state of nematic liquid crystals are described by the minimum of their free energy $F_{t}$ considering the interphase interaction of molecules with the outer walls, impurities and external fields. Of special interest is the magnetic-induced reorientation of NLCs and corresponding threshold values of the external magnetic fields effecting on anisotropic molecules through doped ferromagnetic nanoparticles (FN).

Finding the threshold magnetic field causing the critical elastic deformations of the magnetic doped NLCs is reduced to solving a variational problem for the free energy functional $F_{t}$ composed of the elastic deformation energy and the energy related to the contribution of FNs and the external magnetic field $\boldsymbol{H}$. The latter include interphase interaction of anisotropic molecules with bounding surfaces and the doped FNs, and the interaction of the latters with the external magnetic field $\boldsymbol{H}$. Features of the molecular orientational ordering of the magnetic doped NLCs are manifested in the NLC plano-parallel cell confined by two planar glass surfaces which are mutual spaced on the distance $d$. In the given coordinate framework, the axis $z$ is perpendicular to the glass surfaces, and 
the axis $x$ is directed along the NLC cell. The external magnetic field $\boldsymbol{H}$ is directed along the axis $z$. The total free energy of the system $F_{t}$ includes the above-mentioned distortion energy $F$ and the magnetic energy associated with the influence of FNs and the external magnetic field. The magnetic energy $F_{d}$ in terms of the nematic director $\boldsymbol{n}(\boldsymbol{x})$ and the FN director $\boldsymbol{e}=\boldsymbol{M} / M$ has the form [2]

$$
F^{\prime}=\frac{1}{2} \chi_{a}(\boldsymbol{n} \cdot \boldsymbol{H})^{2}-f M_{s}(\boldsymbol{e} \cdot \boldsymbol{H})-f W(\boldsymbol{e} \cdot \boldsymbol{n})^{2},
$$

where $\chi_{a}$ is the diamagnetic susceptibility anisotropy, $f$ is the FN volume fraction, $M$ is the nanoparticle ferromagnetic magnetization, and $W$ is the density of the binding energy between the FN and the liquid crystal orientations. The first term in (10) is the (direct) anisotropic part of the NLC magnetic energy, the second term represents the dipolar interaction between the FNs and the magnetic field, while the third term is the interaction between FN and NLC. Due to small enough concentration of FNs, the exchange interaction between them is neglected. It is also assumed that the particles are uniformly distributed within the NLC cell avoiding the effect of particle aggregation and segregation.

The effect of a magnetic field on the liquid crystal matrix in a ferronematic suspension can be regarded as the sum of two distinct phenomena. On the one hand, there is a direct effect, associated with the molecular diamagnetic anisotropy that is described by the second term in (10). On the other hand, there is also an indirect effect, associated with the presence of ferromagnetic nanoparticles. This effect is driven by the third term in (10) and acts on the liquid crystal director as a result of the magnetic-nematic coupling described by the fourth term in this equation.

In the frame of coordinates with the $z$ axis perpendicular to the cell surfaces the NLC director and magnetic directors are characterized by the angles $\theta(z)$. Then, it can be shown that minimizing the free energy functional $F_{t}$ with respect to these angles result in the equation of equilibrium states [3]

$$
\begin{gathered}
\frac{d^{2} \theta}{d z^{2}}\left(1+p \sin ^{2} \theta\right)+\left(\frac{d \theta}{d z}\right)^{2} \frac{p}{2} \sin 2 \theta+\frac{h^{2}-b^{2}}{2} \sin \theta \\
+h b \cos 2 \theta+\omega \sin 2(\psi-\theta)=0, \quad \text { (11a) } \\
h k(\mathrm{~h} \cos \psi-b \sin \psi)-\omega \sin 2(\psi-\theta), \quad(11 \mathrm{~b})
\end{gathered}
$$

where $p=\left(K_{3}-K_{1}\right) / K_{1} \quad\left(K_{1}\right.$ and $K_{3}$ are coefficients of transverse and longitudinal bending, respectively), the magnetic field $H$ is represented by the non-dimensional field $h=H / H_{s}$ $\left(H_{s}=\sqrt{K_{1} / \chi_{a}} / d\right)$ and the bias magnetic field $H_{b}$ is scaled $b=H_{b} / H_{s}$. The threshold Frederiks threshold in the pure NLCs at zero surface pre-tilt and zero bias field occurs at $h=\pi$. 
The FN NLC is characterized by the two dimensionless parameters $k=M f d / \sqrt{\chi_{a} K_{1}}$ and $\omega=W f d^{2} / K_{1}$. The magnetic parameter $k$ characterizes the ratio of the magnetic field effect on the ferroparticles to the direct coupling of the magnetic field with the nematic matrix. Thus $k<1 \mathrm{k}<1$ and $k>1$ correspond respectively to the direct or indirect effects dominating the magnetic properties of the ferronematic system. The parameter $\omega$ is the coupling parameter.

Solving (11a) and (11b) determines the nematic and magnetic director orientation angles $\theta(z)$ and $\psi(z)$, as a function of the external and bias magnetic fields. In the case rigid anchorirng between FN and nematic molecules when the nematic and magnetic directors coincide $(\theta=\psi)$, from (11a) and (11b) can be obtained that the threshold magnetic field $H_{c s}=\pi^{2} K_{1} / M d^{2}$, that for typical physical parameters is about hundred gauss.

For typical values of the constants of elastic deformation and magnetizations, the threshold magnetic field of the orientational transition $H_{c s}$ about tens gauss, that is much less than in the case of direct interaction of the magnetic field with diamagnetic molecules of the pure NLCs crystal, for which the threshold fields are the order of tesla.

\section{MOLECULAR DINAMICS OF FERROMAGNETIC NLCS}

The large enough interaction between FNs in NLCs effects on the molecular ordering dynamics. In this case, the magnetic doped NLCs possess two order parameters giving rise to preferred directions $\boldsymbol{n}$ of the average orientation of NLC crystalline molecules and the spontaneous magnetization $\boldsymbol{M}$ (describing the density of magnetic moments of FNs) [3]. As a consequence, optical and magnetic responses are coupled in these materials, which makes them particularly interesting in the multiferroic context: optical properties can be manipulated with a weak external magnetic field (a strong magneto-optic effect) and conversely, the spontaneous magnetization can be reoriented by an external electric field (the converse magnetoelectric effect). The ferromagnetic ordering was observed in the the FN NLCs with platelet-like ferromagnetic particles.

In the studied suspension of magnetic nanoplatelets in the NLC the surface of the nanoplatelets was treated so that they orient with their short axis parallel to $n$. Magnetic interaction between the platelets was such that the magnetic moments of the particles were aligned ferromagnetically, which resulted in a macroscopic $\boldsymbol{M}$ along $\boldsymbol{n}$. The NLC cells in which a liquid crystal is placed between two glass plates with surfaces treated so that in the absence of external fields $\boldsymbol{n}$ and $\boldsymbol{M}$ are homogeneously oriented in the plane of the cell. If an external field is applied perpendicular to $n$, 
both order parameters vary continuously with the position, so that (at constant temperature, pressure, $\boldsymbol{H}$, and the electric displacement field $\boldsymbol{D}$ ) the total free energy $F_{t o t}$ can be written as the $\operatorname{sum} F_{\text {tot }}=\sum_{i} F_{i}$ [3] containing besides the above mentioned the elastic energy $F_{1}=F_{e}$ the energies

$$
F_{2}=-\frac{1}{2} \int_{0}^{d} \gamma \mu_{0}(\boldsymbol{n} \cdot \boldsymbol{M}) d x, F_{3}=-\int_{0}^{d}\left(\mu_{0} \boldsymbol{M} \cdot \boldsymbol{H}+\frac{1}{2} \boldsymbol{D} \cdot \boldsymbol{E}\right) d x
$$

(where $\gamma$ is the coupling constant) related to the coupling magnetization with nematic ordering parameters, and the coupling these ordering parameters with external fields, respectively. Here $\boldsymbol{D}=\varepsilon_{0} \boldsymbol{\varepsilon} \boldsymbol{E}$ and the dielectric tensor $\boldsymbol{\varepsilon}=\varepsilon_{\perp} I+\varepsilon_{a} \boldsymbol{n} \otimes \boldsymbol{n}$, where $\varepsilon_{\perp}$ is the dielectric constant perpendicular to the director $\boldsymbol{n}$ and $\varepsilon_{a}$ is the dielectric anisotropy. The energy $F_{\text {tot }}$ also includes the energy $F_{4}=-W\left(\boldsymbol{n} \cdot \boldsymbol{n}_{s}\right) / 2$ related to the anchoring the NLC directors in the bulk $\boldsymbol{n}$ and on the cell surface $\boldsymbol{n}_{s}$.

Solving the variational problem for the above mentioned free energy functional have permitted to study the response of the ferromagnetic liquid crystal to external magnetic and electric fields, and to compare it to the usual response of the pure NLC. It was shown the existence of effects, which are not present in the pure NLCs and are a consequence of the coupling between the nematic director and the magnetization.

The electro-optic effect, which is in the ferromagnetic phase, the same as in the pure NLC, is accompanied by a converse magneto-electric effect. The magneto-optic effect differs completely from the one observed in the pure NLCs, where it is a quadratic effect and it only appears when a magnetic field larger than a threshold field is applied perpendicular to the director. In the ferromagnetic NLC there are two types of magneto-optic effects. In addition to the response to the field perpendicular to the director, there is also a qualitatively different response to the parallel field. Contrary to the pure NLC no threshold field needs to be exceeded for the system to respond to a field applied perpendicularly to the director, but a threshold field needs to be exceeded to observe a response from the NLCs to the field applied parallel to the director and antiparallel to magnetization. The threshold field is in this case two orders of magnitude smaller than the threshold field of the magnetic Frederiks transition in the pure NLCs.

These ferromagnetic NLCs is so a rather unique systems, in which the magnetization can be controlled by an external electric field, that forms the basis for their possible applications. 


\section{MEASUREMENTS OF MAGNETO-OPTIC EFFECT}

The magneto-optical effect of the influence of a magnetic field (up to $18.6 \mathrm{kOe}$ ) on the light transmission through the NLC cells without and with FNs (based on the compound $\mathrm{Fe}_{2} \mathrm{O}_{3}$ ) has experimentally studied at room temperatures. The polarized light of different frequency was transmitted through the plano-parallel NLC cells of a micrometer thickness with two bounding glass substrates. The internal surfaces of these substrates were covered by the thin conductive transparent layer (on the base of compounds $\mathrm{SiO}_{2}$ ) covered with the ordering layer providing the molecular orientational ordering NLC. This layer, composing of polyamide lacquer or polyvinyl alcohol, was characterized by strong enough anchoring with anisotropic molecules that together with a special microrelief set the molecular orientational ordering.

The magneto-induced optical activity, i.e., the polarization twisting, of the NLCs was investigated by measuring the intensity $I$ of the linearly polarized light transmitted through the NLC cells with and without FNs. This intensity determines is related with the phase difference $\varphi_{d}=\varphi(z)_{z=d}$ between the ordinary and extraordinary output light by the relation [1] $I=I_{0} \sin ^{2} 2 \beta \sin ^{2}\left(\varphi_{d} / 2\right)$, where $\beta$ is the angle between an initial polarization vector and the initial NLC director.

The values of $\varphi(z)$ along the coordinate $z$ is determined by the difference between the refractive indexes $n_{e}(\theta(\mathrm{z}))$ (depending on the angle $\theta(z)$ between the NLC director and the axis $z$ ) and $n_{0}$ of the extraordinary and ordinary light, respectively, i.e., the optical anisotropy $\Delta n(z)=n_{e}(\theta(z))-n_{0}$.

The phase difference of output light is determined through the optical anisotropy $n_{a}=\int_{0}^{d} d z \Delta n(z)$ by the equation as $\varphi_{d}=k_{0} n_{a}$, where $k_{0}=2 \pi / \lambda$ is the wave vector of the monochromatic light wave with the wavelength $\lambda$. The phase difference changes of the transmitted light under an external field (electric or magnetic) are caused by the corresponding change of the optic anisotropy $n_{a}$ depending on the optic axis coinciding with the NLC director.

The investigated magneto-optic effect of the polarization twisting of the transmitted light is caused by the magneto-induced distortion of the NLC director and coincident with it optical axis. Comparison of the magneto-optic effects in NLC cells without and with FNs shown strong influence of the latter on the optical activity of the NLC cells. The observed large magneto-optic effect for the FN doped NLCs manifested in the large polarization twisting angle of about ten degree that makes it possible to create large-format effective magnetic field sensors based on the FN-doped NLCs. 


\section{ACKNOWLEDGMENT}

This work is part of a project that has received funding from the European Union's Horizon 2020 research and innovation programme under the Marie Skłodowska-Curie grant agreement No 778308.

\section{REFERENCES}

[1] .K. Yang, S.T. Wu, Fundamentals of Liquid Crystal Devices, Wiley, West Sussex, 2006.

[2] N. Podoliak, O. Buchnev, O. Buluy, G. D. Alessandro, M. Kaczmarek, Y. Reznikov, and T. J. Sluckinb, "Macroscopic optical effects in low concentration ferronematics," Soft Matter, vol. 7, pp. 4742-4749, March 2011.

[3] H. Pleiner, and D. Svenšek, N. Sebastián, N. Osterman, D. Lisjak, H. R. Brand, H. Pleiner, and D. Svenšek, "Magneto-optic dynamics in a ferromagnetic nematic liquid crystal," Phys. Rev., vol. E 97, pp. 012701(1)-(18), January 2018.

[4] J. Han, Y. Luo, W. Wang, P. Zhang, and Z. Zhang, T. "Scientist, From microscopic theory to macroscopic theory: a systematic study on modeling for liquid crystals," Archive for Rational Mechanics and Analysis, vol. 215, pp. 741-809, April 2014. 